https://doi.org/10.7203/Normas.1.4650

\title{
ANÁLISIS DE HÁBITOS ORTOTIPOGRÁFICOS DEL ALUMNADO UNIVERSITARIO: ORTOGRAFÍA DE LOS ELEMENTOS TIPOGRÁFICOS*
}

\author{
ANALYSIS OF UNIVERSITY STUDENTS TYPOGRAPHICAL HABITS: ORTHOGRAPHY \\ OF TYPOGRAPHIC ELEMENTS
}

Esteban T. MONTORO DEL ARCO

Universidad de Granada

\section{RESUMEN:}

En este trabajo se examinan los hábitos tipográficos de un grupo de estudiantes universitarios, a través de un corpus textual formado por trabajos académicos. Nuestro objetivo es identificar los errores cometidos en cuanto a la ortografía de los elementos tipográficos: particularmente, en lo que respecta a la justificación de líneas, el sangrado, el tipo de párrafo utilizado, el interlineado, el empleo de signos ortográficos en títulos y epígrafes y la localización de los datos de identificación del autor del trabajo. Nuestro análisis muestra un gran desconocimiento de la ortotipografía por parte del alumnado y constituye por ello un punto de partida para futuras acciones docentes que contribuyan a mejorar la redacción de textos académicos en el ámbito universitario.

PALABRAS CLAVE: ortografía, tipografía, ortotipografía, puntuación, norma lingüística.

\section{ABSTRACT:}

The present article focuses on typographical habits of a group of university students by using a corpus based on academic essays. Our goal is to identify common mistakes among students related to orthography of typographic elements, particularly regarding justification, indentation and spacing, type of paragraph used, use of punctuation marks in titles and headings and place where the author's name is included. Our analysis leads to the conclusion that students are largely unaware of typographical rules. Thus, it represents a starting point for future teaching actions in order to improve writing of academic texts at university levels.

KEY WORDS: orthography, typography, typographical syntax, punctuation, linguistic norm.

* Este trabajo se enmarca en el Proyecto de Innovación Docente "Recursos para la enseñanza transversal de la ortografía y la ortotipografía del español” (Ref. 10-156), financiado por el Programa de Innovación y Buenas Prácticas Docentes para el curso académico 2010-2011 de la Universidad de Granada.

NORMAS. REVISTA DE ESTUDIOS LINGÜÍSTICOS HISPÁNICOS, NÚMERO 1 (AÑO 2011):

https://www.uv.es/normas 


\section{INTRODUCCIÓN}

La rápida generalización que ha experimentado la escritura informática, merced a las facilidades que ofrecen los programas de tratamiento de textos, ha propiciado que la inmensa mayoría de los hablantes se familiarice implícitamente con la tipografía técnica, de modo que ésta ha dejado de ser una materia específica de tipógrafos y editores. Sin embargo, el progresivo aumento de las opciones tipográficas que ofrecen los procesadores de textos que utiliza el usuario no tipógrafo, así como el escaso conocimiento de éste con las normas explícitas que guían la elección de las más apropiadas, hace que los textos finales presenten múltiples incongruencias y errores.

Resulta revelador que algunas de las principales y más completas obras de referencia sobre usos normativos y composición textual (por ejemplo, Montolío 2000 y Aleza, coord., 2010) no incluyan apartados dedicados a la ortotipografía. La divulgación de normas ortotipográficas relativas al español es, en realidad, muy reciente. Tuvo un primer gran hito en el manual de Martínez de Sousa (2004), titulado Ortografía y ortotipografía del español actual, pero la fuente de la que emana oficialmente la norma del español, la Real Academia Española, no se ha pronunciado sobre este particular hasta la última edición de su Ortografía de la lengua española (RAE 2010), en la que por primera vez regula algunos aspectos relativos a la ortotipografía, tales como la elección del tipo de fuente (cursiva, negrita, redonda, versal, etc.). A juicio de algunos tipógrafos, no obstante, con ello la Academia excede sus competencias ${ }^{1}$. En cualquier caso, vengan de la mano de tipógrafos o de ortógrafos, lo cierto es que los hablantes necesitamos disponer de unas normas ortotipográficas básicas que garanticen la correcta composición de textos, pues de esta depende también la imagen personal y profesional que proyectamos a la sociedad (Montolío 2010).

\section{HIPÓTESIS Y METODOLOGÍA}

Es sabido que cada texto tiene sus propias convenciones ortotipográficas. En los últimos tiempos el alumnado universitario ha encontrado en Internet una de sus principales fuentes de información. Esto hace que importe de la red una serie de modelos textuales cuyas normas tipográficas -en el caso de que mantengan alguna disciplina en este aspecto- contrastan con las que se deben aplicar en textos de carácter académico (que se hallan implícitas en la mayor parte de los manuales y demás fuentes de información en papel), especialmente en aquellos que han de entregar como resultado de su aprendizaje (informes, resúmenes, etc.) y que constituyen, a su vez, un

1 «La ortotipografía, como su propio nombre indica, atañe a los tipógrafos, no a los académicos. Pese a ello, la obra [la Ortografía de la RAE] está sembrada de datos o apuntes de ortotipografía, datos o apuntes que solo sirven para distraer la atención y cuyo contenido normalmente es innecesario o irrelevante (muchas veces solo contribuye a hacer el texto más amazacotado)» (Martínez de Sousa 2010: 2). 
objeto de evaluación por parte del profesorado. La norma ortotipográfica sigue siendo, en definitiva, una de las grandes asignaturas pendientes de las enseñanzas lingüísticas.

Con objeto de contribuir a la mejora de los hábitos tipográficos de nuestros alumnos, nos propusimos realizar un diagnóstico de los problemas que desde hace tiempo observamos en sus composiciones. Nos interesaba averiguar no solo el tipo de errores que se cometen, sino su extensión e importancia desde un punto de vista cuantitativo y, por ende, cualitativo.

Para la obtención de la muestra nos servimos del sistema de evaluación de la decimosexta edición de un encuentro científico que se desarrolla anualmente en la Facultad de Filosofía y Letras de la Universidad de Granada, las Jornadas sobre la lengua española y su enseñanza, celebradas durante los días 24 a 27 de noviembre de 2010. Esta actividad se integra en el apartado de formación de algunas asignaturas en particular y se ofrece también en general al alumnado de distintos niveles (de primer, segundo y tercer ciclo) relacionado fundamentalmente con las titulaciones de corte humanístico, y más concretamente filológico. El asistente que desee convalidar el curso como créditos de libre elección o como actividad extracurricular $-\mathrm{y}$, además, obtener una calificación- ha de entregar a los organizadores a través del correo electrónico un resumen de una de las sesiones celebradas durante el encuentro (ya sea una conferencia, un taller o una mesa redonda). Los asistentes no recibieron directrices sobre la presentación de dicho trabajo, con objeto de asegurarnos de que cada uno eligiera sus opciones libremente y mostrara sus hábitos en la presentación formal de trabajos académicos breves. Tan solo se les pidió que fueran elaborados con las versiones 2003 o 2007 del procesador de textos Microsoft Office Word ${ }^{\circledR}$.

El corpus textual resultante está integrado, en primer lugar, por 52 trabajos enviados por 52 alumnos distintos de primer curso del Grado en Traducción e Interpretación de la Universidad de Granada, para quienes la asistencia al menos a una de las sesiones fue obligatoria, dado que formaba parte de las actividades presenciales de la asignatura de Lengua Al (Español) del curso 2010/2011: este grupo será denominado "Grupo A". Asimismo, consideramos un grupo heterogéneo, de control, que comprende 23 textos de otros tantos asistentes, procedentes de diversas titulaciones y cursos o niveles de la Facultad de Filosofía y Letras ("Grupo B"). Finalmente, hemos denominado "Grupo A+B" al grupo formado por los dos grupos anteriores y que contiene un total de 75 textos. Nuestra hipótesis de partida consiste en que el grupo A manifestará un mayor porcentaje de usos erróneos, dada su mínima interacción con la tipología textual universitaria, mientras que el grupo B debería haber adquirido mejores hábitos ortotipográficos en virtud de su mayor experiencia y del efecto sancionador del profesorado a lo largo de su formación académica.

Aunque hay múltiples aspectos en este corpus susceptibles de un análisis pormenorizado, para este estudio nos hemos fijado en algunas variables que pertenecen a la llamada ortografía de los elementos tipográficos. Los elementos tipográficos son «las representaciones gráficas o asimiladas (como los espacios en blanco) que pueden individualizarse y considerarse por sí mismas como partes de un todo» (Martínez de Sousa 2004: 399), esto es: los caracteres o grafías, los signos ortográficos o de puntuación, las líneas o renglones, los adornos, etcétera. 
Nos ocuparemos, en primer lugar (\$2), del uso de signos ortográficos (como el punto o los dos puntos) en títulos y epígrafes y, en segundo lugar ( $\$ 3)$, nos detendremos en la grafía de las líneas y los párrafos, así como en la alineación y el espaciado. A continuación, incluiremos algunas observaciones sobre la inclusión de los datos de identificación del alumno en su trabajo (\$4). Finalmente (\$5), haremos una valoración global de los resultados obtenidos y extraeremos las conclusiones oportunas. Para la codificación y análisis de los datos, hemos utilizado el paquete estadístico SPSS ${ }^{\circledR}$.

\section{USO DE SIGNOS ORTOGRÁFICOS EN TÍTULOS Y EPÍGRAFES}

2.1. Uno de los errores tipográficos más frecuentes y llamativos en las composiciones escritas de nuestro alumnado es el uso del punto para cerrar cualquier tipo de título, epígrafe o sub-epígrafe, a pesar de que la norma ha sido siempre clara a este respecto: «el punto se omite en los títulos y subtítulos, cualquiera que sea el lugar en que aparezcan (publicidad, cubiertas y portadas de libros, capítulos artículos de periódicos, etcétera), estén centrados o marginados» (Martínez de Sousa 2004: 304). El ejemplo siguiente pertenece a un sujeto que comete este error tanto en el título como en el epígrafe y sub-epígrafe que le siguen ${ }^{2}$ :

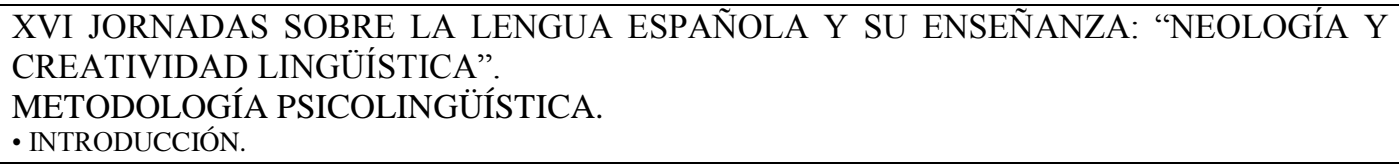

El uso de los dos puntos (:) tampoco ha de utilizarse para este fin, pero también se encuentra documentado en el corpus:

\section{Mi conclusión y opinión personal sobre el taller:}

Por una parte, encuentro que este taller puede ser de utilidad para conocer cómo nuestro cerebro maneja el proceso de comprensión de un lenguaje, ya que somos estudiantes de Traducción e Interpretación, y por lo tanto vamos a desenvolvernos en un ambiente idiomáticamente diverso. Por otra parte, creo que éste taller les sería de más utilidad a aquellas personas que estudien Pedagogía o para aquellos que estudien Psicología orientada al lenguaje.

Dado que no se dieron pautas para la redacción de los textos, no todos los informantes incluyeron títulos y epígrafes. Por tanto, si descartamos los textos en los que estos no aparecen, observamos (tabla 1), que el uso de dos puntos en los títulos es prácticamente anecdótico, pero no así el del punto, que supera un tercio de la muestra en el grupo A e incluso se acerca a la mitad del grupo B:

${ }^{2}$ Los textos originales que se presentan como ejemplos se transcriben respetando el formato de origen, excepto en el tamaño de fuente.

NORMAS. REVISTA DE ESTUDIOS LINGÜÍSTICOS HISPÁNICOS, NÚMERO 1 (AÑO 2011): https://www.uv.es/normas 
Tabla 1. Título. Frecuencias absolutas

\begin{tabular}{|c|c|c|c|c|c|c|}
\cline { 2 - 7 } \multicolumn{1}{c|}{} & \multicolumn{2}{c|}{ Grupo A } & \multicolumn{2}{c|}{ Grupo B } & \multicolumn{2}{c|}{ Grupo A+B } \\
\cline { 2 - 7 } \multicolumn{1}{c|}{} & $\mathrm{N}$ & $\%$ & $\mathrm{~N}$ & $\%$ & $\mathrm{~N}$ & $\%$ \\
\hline Sin punto & 30 & 61.2 & 11 & 55.0 & 41 & 59.4 \\
\hline Con punto & 18 & 36.7 & 9 & 45.0 & 27 & 39.1 \\
\hline Dos puntos & 1 & 2.0 & 0 & 0.0 & 1 & 1.4 \\
\hline TOTAL & 49 & 100.0 & 20 & 100.0 & 69 & 100.0 \\
\hline
\end{tabular}

En el caso de los epígrafes, el uso del punto mantiene unas cifras similares, pero aumenta el porcentaje de empleo de los dos puntos (tabla 2):

Tabla 2. Epígrafes. Frecuencias absolutas

\begin{tabular}{|c|c|c|c|c|c|c|}
\multicolumn{1}{c|}{ Tabla 2. Epígrafes. Frecuencias absolutas } \\
\cline { 2 - 7 } \multicolumn{1}{c|}{ Grupo A } & \multicolumn{2}{c|}{ Grupo B } & \multicolumn{2}{c|}{ Grupo A+B } \\
\cline { 2 - 7 } & $\mathrm{N}$ & $\%$ & $\mathrm{~N}$ & $\%$ & $\mathrm{~N}$ & $\%$ \\
\hline Sin punto & 13 & 46.4 & 6 & 46.1 & 19 & 46.3 \\
\hline Con punto & 10 & 35.7 & 5 & 38.5 & 15 & 36.6 \\
\hline Dos puntos & 5 & 17.9 & 2 & 15.4 & 7 & 17.1 \\
\hline TOTAL & 28 & 100.0 & 13 & 100.0 & 41 & 100.0 \\
\hline
\end{tabular}

Como se ha indicado, el uso de un signo ortográfico para cerrar un título (ya sea punto o dos puntos) representa un error frente a la opción correcta, que es no usar signo alguno. Tras asimilar el uso de los dos puntos al del punto para obtener una oposición binaria (normativo/antinormativo), hemos procedido a comparar los dos grupos (A frente a B). Así, observamos una diferencia significativa en el empleo de signos ortográficos en los títulos por parte del grupo A (tabla 4), en el que hasta un $71,4 \%$ incurre en dicho error (frente al 35\% del grupo B):

Tabla 3. Uso de signo ortográfico (títulos)

\begin{tabular}{|c|c|c|}
\cline { 2 - 3 } \multicolumn{1}{c|}{} & $\mathrm{N}$ & $\%$ \\
\hline Grupo A & $35 / 49$ & 71.4 \\
\hline Grupo B & $7 / 20$ & 35.0 \\
\hline TOTAL & $42 / 69$ & 60.9 \\
\hline \multicolumn{3}{|c|}{$\mathrm{Chi}^{2}=7.913 \quad$ Sig. 005 } \\
\hline
\end{tabular}

En cambio, comprobamos que el uso de signos ortográficos en los epígrafes no diferencia mucho a los dos grupos, aunque lo cierto es que, desde un punto de vista normativo, en ambos casos los porcentajes son muy elevados y, por ende, preocupantes:

Tabla 4. Uso de signo ortográfico (epígrafes)

\begin{tabular}{|c|c|c|}
\cline { 2 - 3 } \multicolumn{1}{c|}{} & $\mathrm{N}$ & $\%$ \\
\hline Grupo A & $19 / 49$ & 38.8 \\
\hline Grupo B & $9 / 20$ & 45.0 \\
\hline TOTAL & $28 / 69$ & 40.6 \\
\hline \multicolumn{3}{|c|}{$\mathrm{Chi}^{2}=228$ Sig. 633 } \\
\hline
\end{tabular}


2.2. Llegados a este punto, aún cabe preguntarse cuántos de los sujetos estudiados evitan el uso de signos ortográficos tanto en el título como en los epígrafes dentro de un mismo texto. Para ello, hemos de descartar los casos en los que el sujeto no incluye epígrafes (pues este hecho no es un error en sí mismo), aunque consideraremos que el hecho de no incluir título sí representa un error, dado que el título sí constituye un rasgo fundamental del género discursivo al que pertenecen estos textos. Nos quedamos, pues, con 41 casos de 75 en el caso del grupo amplio $(A+B)$, y 28 de 52 en el caso del grupo A.

Al cruzar los casos de la variable "título" con las de la variable "epígrafes" (tabla 5), podemos comprobar qué porcentaje de sujetos cumple al mismo tiempo con ambos criterios de corrección y, por consiguiente, compone bien su texto desde este punto de vista. En el caso del grupo A, tan solo un $35,7 \%$ de los sujetos no hace uso de los signos ortográficos en títulos ni epígrafes:

Tabla 5. Uso de signos ortográficos en títulos y epígrafes (Grupo A)

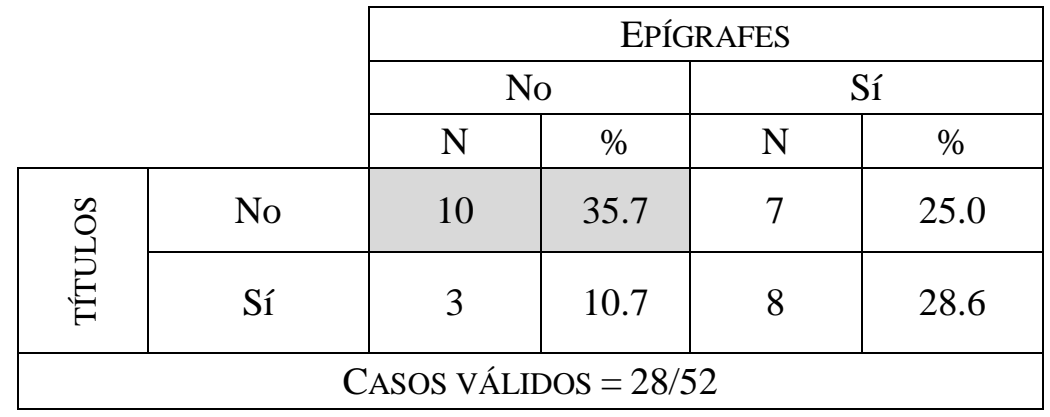

Los datos son muy similares si consideramos todo el grupo $(A+B)($ tabla 6): tan solo 15 alumnos (que representan un 36,6\% del total) dispone correctamente títulos y epígrafes en su escrito. En el otro extremo, casi el mismo número (14, un 34,1\%) yerra tanto en títulos como en epígrafes; y un $29,3 \%$ comete errores en uno u otro caso:

Tabla 6. Uso de signos ortográficos en títulos y epígrafes $(\mathrm{A}+\mathrm{B})$

\begin{tabular}{|c|c|c|c|c|c|}
\hline & & \multirow{2}{*}{\multicolumn{4}{|c|}{ EPÍGRAFES }} \\
\hline & & & & & \\
\hline & & \multicolumn{2}{|c|}{ No } & \multicolumn{2}{|c|}{ Sí } \\
\hline & & $\mathrm{N}$ & $\%$ & $\mathrm{~N}$ & $\%$ \\
\hline \multirow{2}{*}{ 。 } & No & 15 & 36.6 & 8 & 19.5 \\
\hline & Sí & 4 & 9.8 & 14 & 34.1 \\
\hline \multicolumn{6}{|c|}{ CASOS VÁLIDOS $=41 / 75$} \\
\hline
\end{tabular}

En general, el porcentaje de casos incorrectos es extremadamente elevado $(64,3 \%$ en el grupo A y $64,4 \%$ en el grupo A+B). Puesto que la norma es clara en el caso del español, hemos de pensar que el profesorado no incide lo suficiente en la corrección de 
este fenómeno y que el alumnado no conoce la norma o no es capaz de inferirla a partir de las fuentes bibliográficas que maneja.

\section{LAS LÍNEAS Y LOS PÁRRAFOS}

En el marco de la grafía de la línea (Martínez de Sousa 2004) o formato de la línea (Almela 2010), varios son los apartados que han llamado nuestra atención: la justificación de las líneas, las líneas sangradas, el espaciado y el interlineado, así como el tipo de párrafo utilizado (inherente a la consideración de los aspectos anteriores, como vamos a ver).

\subsection{Justificación de líneas}

De forma general, el número de caracteres que incluye un renglón o línea no suele coincidir con el del resto, puesto que no es igual el número de palabras que los forman y porque unos renglones están sangrados y otros no, como se puede observar en el siguiente ejemplo (tabla 7):

Tabla 7. Irregularidad de las líneas

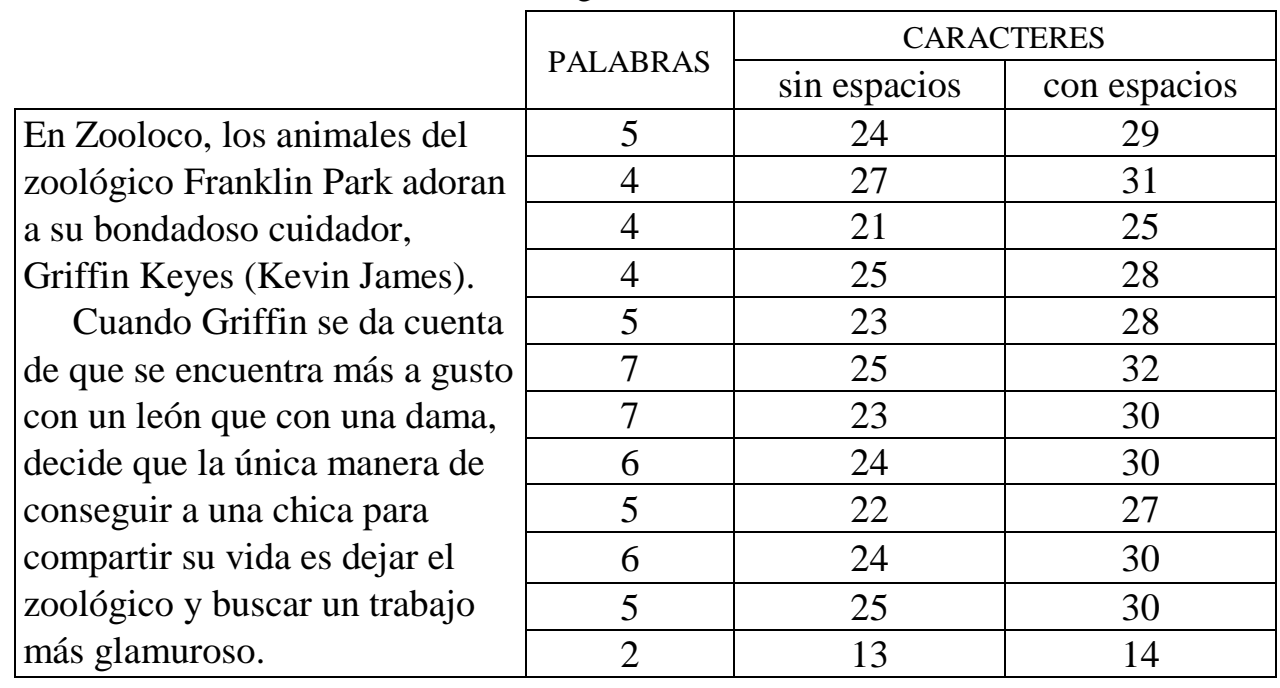

La diferencia en el número de caracteres que conforma cada línea hace que el texto en conjunto resulte asimétrico, en tanto que no coincide exactamente por la parte derecha con los límites de la caja de escritura -espacio limitado por renglones y márgenes-. Este tipo de alineación es solo una de las cuatro que, en general, suelen tener los textos (tabla 8): 
Tabla 8. Tipos de alineación

\begin{tabular}{|c|c|c|c|}
\hline IZQUIERDA & DERECHA & CENTRADA & JUSTIFICADA \\
\hline $\begin{array}{l}\text { En Zooloco, los } \\
\text { animales del } \\
\text { zoológico Franklin } \\
\text { Park adoran a su } \\
\text { bondadoso cuidador, } \\
\text { Griffin Keyes (Kevin } \\
\text { James). }\end{array}$ & $\begin{array}{r}\text { En Zooloco, los } \\
\text { animales del } \\
\text { zoológico Franklin } \\
\text { Park adoran a su } \\
\text { bondadoso } \\
\text { cuidador, Griffin } \\
\text { Keyes (Kevin } \\
\text { James). }\end{array}$ & $\begin{array}{c}\text { En Zooloco, los } \\
\text { animales del } \\
\text { zoológico Franklin } \\
\text { Park adoran a su } \\
\text { bondadoso } \\
\text { cuidador, Griffin } \\
\text { Keyes (Kevin } \\
\text { James). }\end{array}$ & $\begin{array}{l}\text { En Zooloco, los } \\
\text { animales } \\
\text { zoológico Franklin } \\
\text { Park adoran a su } \\
\text { bondadoso } \\
\text { cuidador, } \\
\text { Keyes } \\
\text { James). }\end{array}$ \\
\hline
\end{tabular}

A veces las elecciones del usuario dependen de gustos personales o de la mera costumbre. No obstante, cada una de estas alineaciones tiene su función y por ello están asociadas a diferentes tipos textuales. Así, una alineación totalmente asimétrica, como la centrada, resulta chocante para el lector, pero dota al texto de una gran expresividad. En los textos de tipo académico, en cambio, se recomienda la alineación justificada, pues se consigue el efecto contrario: el texto adquiere una apariencia seria y solemne acorde con el ámbito en el que es producido y consumido. Para conseguir este tipo de alineación armónica se hace necesaria una operación que se denomina justificación, consistente en «aumentar o disminuir el espacio normal entre palabras, con lo que se consigue que el texto de cada línea llegue exactamente hasta su final» (Martínez de Sousa 2004: 402) o, lo que es lo mismo, en alinear el texto a derecha e izquierda. Esta operación se realiza automáticamente en los procesadores de textos al uso, que incorporan además la opción de introducir guiones al final del renglón para que el espacio entre los caracteres no sea excesivo (véase más arriba la tabla 8):

En Zooloco,, los animales del zoológico Franklin Park adoran a su bondadoso cuidador, Griffin Keyes (Kevin James). Cuando Griffin se da cuenta de que se encuentra más a gusto con un león que con una dama, decide que la única manera de conseguir a una chica para compartir su vida es dejar el zoológico y buscar un trabajo más glamuroso. Los animales entran en pánico y DECIDEN romper el código que han mantenido desde siempre y revelar su mayor secreto: ¡pueden hablar! Para evitar que Griffin se vaya, deciden enseñarle las reglas del cortejo al más puro estilo animal. La película también cuenta con la presencia de Rosario Dawson y Leslie Bibb y con las voces de Cher, Nick Nolte, Adam Sandler y Sylvester Stallone.

En Zooloco,, los animales del zoológico Franklin Park adoran a su bondadoso cuidador, Griffin Keyes (Kevin James). Cuando Griffin se da cuenta de que se encuentra más a gusto con un león que con una dama, decide que la única manera de conseguir a una chica para compartir su vida es dejar el zoológico y buscar un trabajo más glamuroso. Los animales entran en pánico y DECIDEN romper el código que han mantenido desde siempre y revelar su mayor secreto: ¡pueden hablar! Para evitar que Griffin se vaya, deciden enseñarle las reglas del cortejo al más puro estilo animal. La película también cuenta con la presencia de Rosario Dawson y Leslie Bibb y con las voces de Cher, Nick Nolte, Adam Sandler y Sylvester Stallone.

Sin embargo, la mayor parte de los textos que encontramos en Internet no están justificados, pues se entiende que la alineación izquierda es el formato de mayor legibilidad para este medio. La norma de los textos digitales ofrece, pues, un modelo 
diferente del que ha de emplearse en la redacción de otro tipo de textos, como los académicos que forman nuestro corpus. En este, el número de textos no justificados es muy superior al del número de textos justificados (tabla 9):

Tabla 9. Justificación. Frecuencias absolutas (Grupo A+B)

\begin{tabular}{|c|c|c|}
\cline { 2 - 3 } \multicolumn{1}{c|}{} & $\mathrm{N}$ & $\%$ \\
\hline Justificado & 29 & 38.7 \\
\hline No justificado & 44 & 58.7 \\
\hline Mezcla & 2 & 2.7 \\
\hline TOTAL & 75 & 100.0 \\
\hline
\end{tabular}

Al analizar la distribución por grupos de los textos mal alineados (sin justificación a la derecha o con mezcla de distintos tipos de justificación), encontramos que es mucho mayor el porcentaje de casos en el grupo de alumnos recién llegados a la universidad (grupo A) que en el grupo de control (grupo B) (véase tabla 10):

Tabla 10. Textos no justificados

\begin{tabular}{|c|c|c|}
\cline { 2 - 3 } \multicolumn{1}{c|}{} & Tabla 10. Textos no justificados \\
\cline { 2 - 3 } \multicolumn{1}{c|}{} & $\mathrm{N}$ & $\%$ \\
\hline Grupo A & $37 / 52$ & 71.2 \\
\hline Grupo B & $9 / 23$ & 39.1 \\
\hline TOTAL & $46 / 75$ & 61.3 \\
\hline \multicolumn{2}{c}{} \\
\hline
\end{tabular}

Hemos de suponer, por tanto, que el sistema rectifica de algún modo este tipo de error ortotipográfico a lo largo de la etapa universitaria, pero no lo suficiente.

\subsection{Línea sangrada}

3.2.1. La línea sangrada es aquella «que comienza con sangría, es decir, un blanco que se coloca al principio de la primera línea en el párrafo ordinario y en la segunda y siguientes en el párrafo francés» (Martínez de Sousa 2004: 403). Su uso define al llamado párrafo ordinario, esto es, el párrafo de líneas justificadas que comienza con una sangría en la primera línea, como en el siguiente modelo:

Párrafo ordinario párrafo ordinario párrafo ordinario párrafo ordinario párrafo ordinario párrafo ordinario párrafo ordinario párrafo ordinario párrafo ordinario párrafo ordinario párrafo ordinario párrafo ordinario párrafo ordinario párrafo ordinario párrafo ordinario párrafo ordinario párrafo ordinario párrafo ordinario párrafo ordinario párrafo ordinario párrafo ordinario párrafo ordinario párrafo ordinario.

Párrafo ordinario párrafo ordinario párrafo ordinario párrafo ordinario párrafo ordinario párrafo ordinario párrafo ordinario párrafo ordinario párrafo ordinario párrafo ordinario párrafo ordinario párrafo ordinario párrafo ordinario párrafo ordinario párrafo ordinario párrafo ordinario párrafo ordinario párrafo ordinario párrafo ordinario párrafo ordinario párrafo ordinario párrafo ordinario párrafo ordinario.

NORMAS. REVISTA DE ESTUDIOS LINGÜÍSTICOS HISPÁNICOS, NÚMERO 1 (AÑO 2011): https://www.uv.es/normas 
El párrafo ordinario es el más usado en español, así como en un mayor número de tipos textuales. Sin embargo, últimamente se encuentra en muchos textos el denominado párrafo alemán o párrafo moderno, que no lleva sangría alguna en dicha primera línea. Como sugiere Martínez de Sousa (2004: 404), este tipo de composición «es poco práctica en los libros, por cuanto exige separar los párrafos o hacer que doble la línea final del párrafo cuando no resulte normalmente corta». En efecto, la razón de ser de la línea sangrada es marcar visualmente la transición de un párrafo a otro. Si se prescinde del sangrado, ha de utilizarse otro medio que cumpla con la misma función. El más frecuente para ello es la inclusión de una marca de párrafo ${ }^{3}$ entre uno y otro.

Párrafo moderno párrafo moderno párrafo moderno párrafo moderno párrafo moderno párrafo moderno párrafo moderno párrafo moderno párrafo moderno párrafo moderno párrafo moderno párrafo moderno párrafo moderno párrafo moderno párrafo moderno párrafo moderno párrafo moderno párrafo moderno párrafo moderno párrafo moderno párrafo moderno párrafo moderno párrafo moderno párrafo moderno.

Párrafo moderno párrafo moderno párrafo moderno párrafo moderno párrafo moderno párrafo moderno párrafo moderno párrafo moderno párrafo moderno párrafo moderno párrafo moderno párrafo moderno párrafo moderno párrafo moderno párrafo moderno párrafo moderno párrafo moderno párrafo moderno párrafo moderno párrafo moderno párrafo moderno párrafo moderno párrafo moderno párrafo moderno.

El alumnado suele confundir estos dos sistemas, de forma que utiliza, dentro del mismo texto, la línea sangrada y la separación por marca de párrafo. De este modo, se reúnen dos modelos de párrafo en una misma composición y se rompe su coherencia gráfica $^{4}$, como se muestra en el siguiente ejemplo:

Teniendo en cuenta datos anteriores publicados, el investigador puede partir de la idea de que a más temprana edad, más rápido es el procesamiento de esa palabra. Esto sería la hipótesis.

Para verificarla, planifica un experimento en el que los participantes tienen que decidir si las secuencias de letras son palabras o no. Esto sería la tarea de decisión léxica.

Si atendemos al uso de la línea sangrada, los datos revelan que el uso del párrafo ordinario, el característico de la norma española, es minoritario en el grupo A de nuestro corpus:

\footnotetext{
${ }^{3}$ Como es sabido, las marcas de párrafo (resultado de pulsar la tecla "Enter/Intro" de un teclado estándar) son, en principio, transparentes para el usuario, pero vienen representadas en el formato oculto del procesador por el símbolo del calderón: II.

4 “Tal vez por influencia de tipografías extrañas, se ha puesto de moda en la tipografía española prescindir de la sangría en el párrafo ordinario cuando este comienza capítulo o va después de título o subtítulo. [...] al suprimir la sangría del primer párrafo cambiamos de modelo de párrafo, y pasamos de un párrafo ordinario a uno alemán o moderno. Es decir, que componemos o disponemos el texto según dos modelos de párrafo, lo cual no parece bien justificado. La coherencia gráfica pide que en un mismo texto se usen modelos de párrafos iguales" (Martínez de Sousa 2004: 403).
} 
Tabla 11. Línea sangrada. Frecuencias absolutas

\begin{tabular}{|c|c|c|c|c|c|c|}
\hline & \multicolumn{2}{|c|}{ Grupo A } & \multicolumn{2}{|c|}{ Grupo B } & \multicolumn{2}{|c|}{ Grupo $A+B$} \\
\hline & $\mathrm{N}$ & $\%$ & $\mathrm{~N}$ & $\%$ & $\mathrm{~N}$ & $\%$ \\
\hline Línea sangrada & 14 & 26.9 & 6 & 26.1 & 20 & 26.7 \\
\hline Sin sangría & 31 & 59.6 & 14 & 60.9 & 45 & 60.0 \\
\hline Mezcla & 7 & 13.5 & 3 & 13.0 & 10 & 13.3 \\
\hline TOTAL & 52 & 100.0 & 23 & 100.0 & 75 & 100.0 \\
\hline
\end{tabular}

Dado que hasta un $60 \%$ de la muestra no utiliza la línea sangrada, hemos de pensar que quizá utilizan el párrafo alemán. Por ello, hemos de comprobar que la falta de sangrado en la primera línea se corresponda con el uso de otro tipo de marca que indique separación de párrafos, y viceversa: que el uso de la línea sangrada no conlleve separación de párrafos. La tabla 6 recoge la combinación de estas dos variables en el caso del grupo A:

Tabla 12. Tipo de párrafo (Grupo A)

\begin{tabular}{|c|c|c|c|c|c|c|c|}
\hline \multirow{6}{*}{ 岕㐫 } & & \multicolumn{6}{|c|}{ SEPARACIÓN DE PÁRRAFOS } \\
\hline & & \multicolumn{2}{|c|}{ No } & \multicolumn{2}{|c|}{ Sí } & \multicolumn{2}{|c|}{ Mezcla } \\
\hline & & $\mathrm{N}$ & $\%$ & $\mathrm{~N}$ & $\%$ & $\mathrm{~N}$ & $\%$ \\
\hline & Sí & 5 & 9.6 & 7 & 13.5 & 2 & 3.8 \\
\hline & No & 3 & 5.8 & 17 & 32.7 & 11 & 21.2 \\
\hline & Mezcla & 1 & 1.9 & 2 & 3.8 & 4 & 7.7 \\
\hline
\end{tabular}

Esta tabla muestra, en primer lugar, que tan solo un 9,6\% de todos los sujetos del grupo A utiliza el párrafo ordinario y cumple así como la norma establecida, a saber: la conjunción de la línea sangrada y la opción de no separar los párrafos. Recordemos que se trata de 5 alumnos de un total de 52. En segundo lugar, de forma consciente o no, un $32,7 \%$ utiliza el párrafo moderno o alemán, esto es: no sangra la primera línea del párrafo pero marca la transición de uno a otro párrafo con su separación visual a través, bien de la marca de párrafo, bien del espaciado (que, sin embargo, viene en algunos procesadores como opción por defecto). Por último, resulta preocupante que el 57,7 \% de los sujetos del grupo A no tenga criterio alguno al ordenar tipográficamente los párrafos de sus escritos, pues combina de forma inadecuada las variables descritas.

Los resultados del Grupo A+B (tabla 7) son muy similares: 
Tabla 13. Tipo de párrafo (Grupo A+B)

\begin{tabular}{|c|c|c|c|c|c|c|c|}
\hline & & \multicolumn{6}{|c|}{ SEPARACIÓN DE PÁRRAFOS } \\
\hline & & \multicolumn{2}{|c|}{ No } & \multicolumn{2}{|c|}{ Sí } & \multicolumn{2}{|c|}{ Mezcla } \\
\hline \multirow{4}{*}{ 岕定 } & & $\mathrm{N}$ & $\%$ & $\mathrm{~N}$ & $\%$ & $\mathrm{~N}$ & $\%$ \\
\hline & Sí & 6 & 8.0 & 12 & 16.0 & 2 & 2.7 \\
\hline & No & 3 & 4.0 & 23 & 30.7 & 19 & 25.3 \\
\hline & Mezcla & 1 & 1.3 & 3 & 4.0 & 6 & 8.0 \\
\hline
\end{tabular}

3.2.2. Aún queda un aspecto a tener en cuenta en lo que respecta a la línea sangrada. El sangrado de la primera línea es una operación que se puede realizar mediante una herramienta del procesador de textos habilitada para ello (imagen 1):

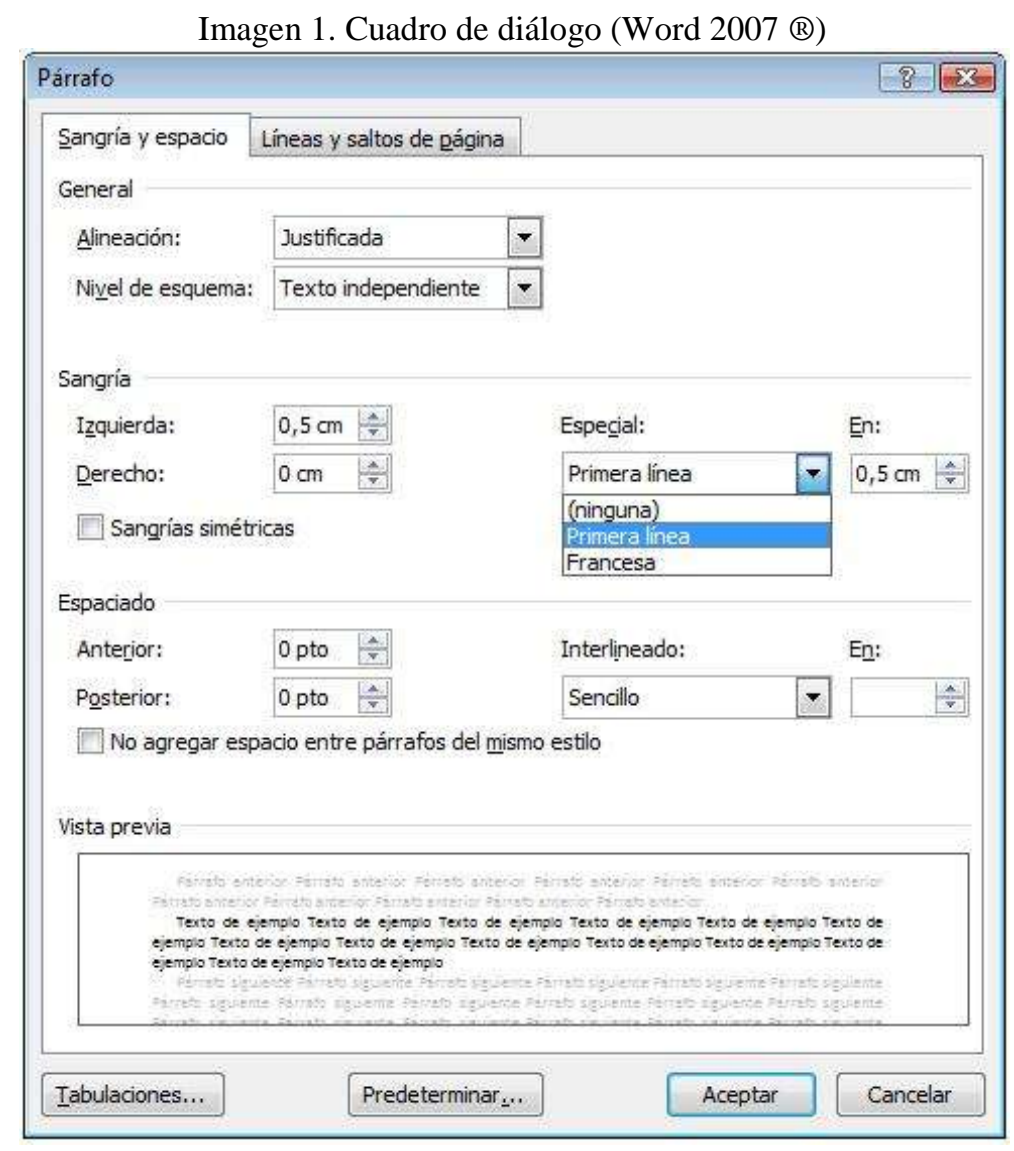

Sin embargo, los sujetos del corpus parecen desconocer esta opción y para conseguir el mismo efecto, al menos en apariencia, recurren al uso de la barra espaciadora del teclado, que va marcando espacios en blanco uno por uno. Como mostramos en la tabla 8, por un lado, la diferencia entre los grupos no es significativa: en principio, parece un hábito que no se corrige necesariamente a lo largo de la etapa 
universitaria; por otro lado, resulta ciertamente alarmante que hasta un $41,3 \%$ de los individuos del corpus incurra en esta práctica errónea:

\begin{tabular}{|c|c|c|} 
Tabla 14. Uso de la barra espaciadora (A+B) \\
\cline { 2 - 3 } & $\mathrm{N}$ & $\%$ \\
\hline Grupo A & $19 / 52$ & 36.5 \\
\hline Grupo B & $12 / 23$ & 52.2 \\
\hline A+B & $31 / 75$ & 41.3 \\
\hline \multicolumn{2}{|c|}{$\mathrm{Chi}^{2}=1.608$ Sig. 205} \\
\hline
\end{tabular}

Pero lo más llamativo es que el uso de la barra afecta también a aquellos alumnos que, en principio, parecen observar un uso correcto del párrafo ordinario: de los 20 alumnos que sangran la primera línea (véase tabla 11), tan solo 13 parecen utilizar correctamente la herramienta del procesador para ello, frente a 7 que recurren al "truco" del uso de la barra espaciadora del ordenador (tabla 9):

Tabla 15. Línea sangrada y uso de la barra espaciadora $(\mathrm{A}+\mathrm{B})$

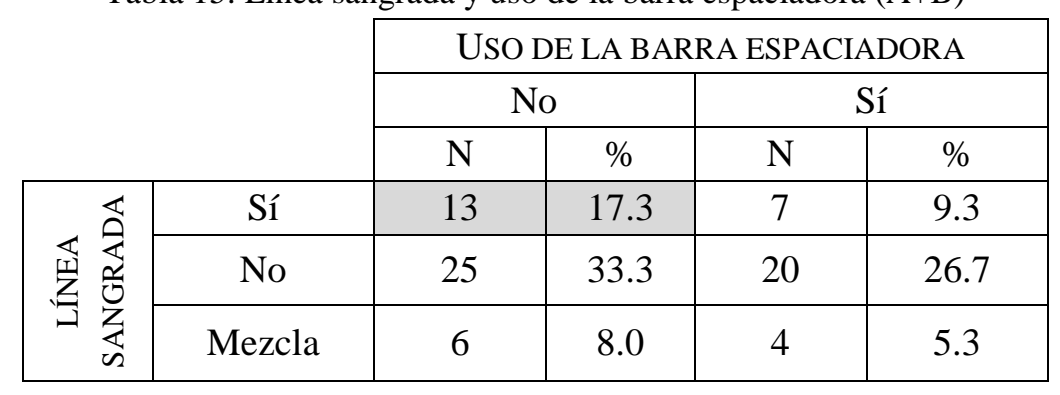

El uso de la barra espaciadora representa, pues, otra práctica inapropiada por parte del alumnado: implica una falta de destreza en el uso del teclado, que exige un tiempo y un esfuerzo totalmente innecesarios. Aparte, no siempre procura el resultado esperado, pues a veces no coincide el número de espacios marcados para conseguir el efecto de sangrado. En el ejemplo siguiente, utilizamos los corchetes para enmarcar los espacios (.) introducidos por su autor a través de la barra espaciadora (espacios que son transparentes para el lector):

[...... La equivalencia entre pronombres puede ser categorial, funcional o pragmática. En las locuciones pronominales personales encontramos un continuo entre dos polos opuestos:

[.....]Ejemplo 1:

[........... Trabajas demasiado

[..........]Tú trabajas demasiado (contraste del tú frente al resto de personas: es muy[..]

$[$............ significativo)

Como puede comprobarse, con gran esfuerzo se intenta conseguir un efecto que el procesador nos permite obtener fácilmente, y sin errores tales como el del número de espacios obtenidos tras pulsar la barra. 


\subsection{Interlineado}

El interlineado (también llamado espaciado entre líneas o regleteado) suele establecerse dependiendo de varios factores que tienen que ver con la correcta legilibilidad del texto, tales como el tamaño de fuente, el tamaño de la página final, etc. En la mayor parte de las hojas de estilo de las revistas especializadas, suele ser de un punto o punto y medio (siendo el tamaño de fuente de 12 puntos). Esta tendencia es también mayoritaria entre nuestro alumnado:

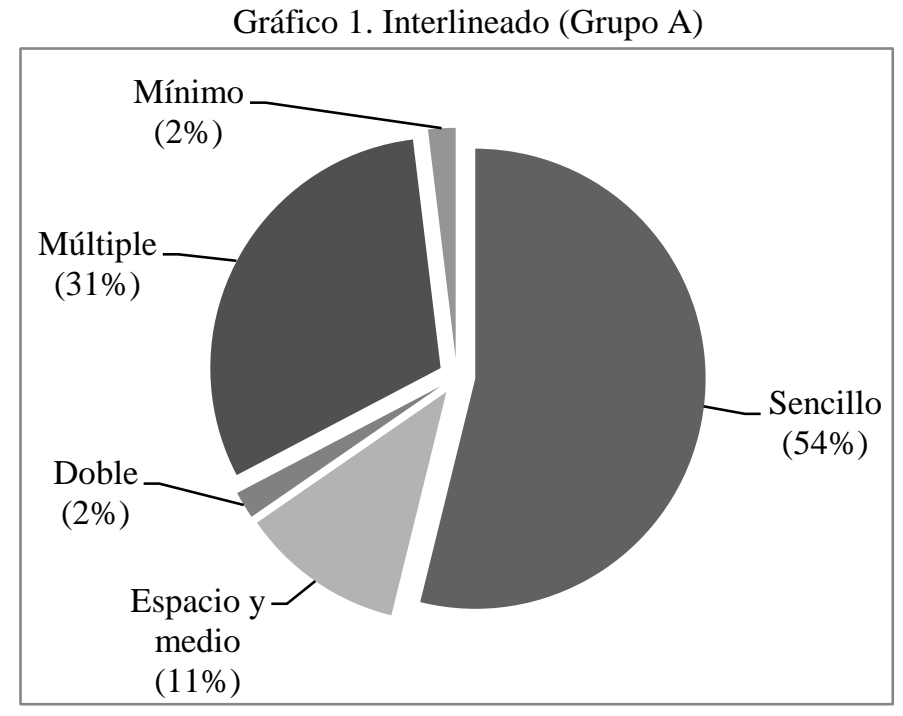

El resultado es prácticamente el mismo si comparamos ambos grupos (tabla 5):

Tabla 16. Interlineado

\begin{tabular}{|c|c|c|c|c|}
\cline { 2 - 5 } \multicolumn{1}{c|}{} & \multicolumn{2}{c}{ Tabla 16. Interlineado } \\
\cline { 2 - 5 } \multicolumn{1}{c|}{ GRUPO A } & $\%$ & $\mathrm{~N}$ & $\%$ \\
\cline { 2 - 5 } \multicolumn{1}{c|}{} & $\mathrm{N}$ & 53.8 & 41 & 54.6 \\
\hline Sencillo & 28 & 11.5 & 9 & 12.0 \\
\hline Espacio y medio & 6 & 1.9 & 1 & 1.3 \\
\hline Doble & 1 & 30.7 & 23 & 30.6 \\
\hline Múltiple & 16 & 1.9 & 1 & 1.3 \\
\hline Mínimo & 1 & 100.0 & 75 & 100.0 \\
\hline TotAL & 52 & & & \\
\hline
\end{tabular}

En este apartado no podemos sino reflejar las actitudes del alumnado, pues es difícil determinar en qué medida el interlineado obedece a una opción personal o se trata de la opción que viene seleccionada por defecto en el procesador de textos utilizado. En 
cualquier caso, sería deseable una norma que proporcionara un modelo de corrección también para este aspecto.

\section{DATOS DE IDENTIFICACIÓN DEL ALUMNO/A}

El último de los fenómenos que estudiamos aquí es el de la correcta identificación del alumno. El siguiente dato resulta bastante sorprendente: hasta 19 alumnos del grupo A no se identificaron como autores del trabajo entregado (tabla 17). Sin duda, este es un problema que trasciende los límites de los hábitos tipográficos que son objeto de nuestro estudio y se sitúa en el ámbito de las prácticas textuales consideradas en sentido amplio.

Se nos ocurren al menos dos explicaciones: en primer lugar, el método de entrega de los trabajos -el correo electrónico-, quizá provoca una cierta relajación, pues el alumno confiaría en que sus datos ya figurasen en algún lugar del mensaje electrónico; sin embargo, se dan casos en los que ni en el archivo adjunto, ni en el cuerpo del mensaje, ni en la firma al final de este, se encuentra el nombre (y tampoco la dirección de correo electrónico ofrece pista alguna). Esta cuestión entronca directamente con el problema de la cortesía en la red o netiqueta (Shea 1994, Millán 2008) y, en particular, con las buenas prácticas en la redacción de correos electrónicos. Las normas de netiqueta son claras a este respecto: como ejemplo, la Open University recalca la siguiente: «Identify yourself - you can use a signature file to put your name at the end of your e-mail automatically, but keep it short (name, title, contact details)».

Tabla 17. Datos de identificación

\begin{tabular}{|c|c|c|c|c|}
\cline { 2 - 4 } \multicolumn{1}{c|}{} & \multicolumn{2}{c|}{ Grupo A } & \multicolumn{2}{c|}{ Grupo B } \\
\cline { 2 - 5 } \multicolumn{1}{c|}{} & $\mathrm{N}$ & $\%$ & $\mathrm{~N}$ & $\%$ \\
\hline Al comienzo & 10 & 19.2 & 11 & 47.8 \\
\hline Al final & 14 & 26.9 & 4 & 17.4 \\
\hline Portada & 1 & 1.9 & 2 & 8.7 \\
\hline Encabezado/pie de página & 8 & 15.4 & 5 & 21.7 \\
\hline Sin datos & 19 & 36.5 & 1 & 4.3 \\
\hline TOTAL & 52 & 100.0 & 23 & 100 \\
\hline
\end{tabular}

En segundo lugar, la existencia de una relación académica previa en el caso del grupo A garantizaba la seguridad de coincidir con el profesor-evaluador tras el evento, lo que permitiría reparar el error en el caso de que este se produjera. Los miembros del grupo $\mathrm{B}$, por el contrario, no tenían una relación directa con el evaluador, de modo que la entrega del trabajo suponía una única oportunidad para justificar la asistencia y para aprobar el curso.

En cualquier caso, no incluir nombre y apellidos en un trabajo personal no deja de ser un error grave, pues es contraproducente para el propio alumno, principal interesado en que su trabajo se evalúe; pero, al mismo tiempo, supone una inconveniencia para la gestión de archivos y datos por parte del evaluador. 
Cuestión distinta es la ubicación correcta de los datos de identificación. Desde el punto de vista del evaluador, la opción más adecuada es obtener los datos al comienzo, no al final, pues facilita la tarea de evaluación, de forma análoga a la práctica habitual en exámenes y pruebas de diversa índole. Algunos alumnos incluyen una portada que quizá resulta innecesaria para este tipo de trabajos, pero que cumple con la función antes descrita: sumando las variantes "al comienzo" y "en portada", tan solo un $21,1 \%$ del grupo A se identifica de forma adecuada.

Desde un punto de vista tipográfico, estos datos han de figurar dentro de la caja de escritura, independientemente de que luego se utilice el encabezado para reflejar algunos de estos datos (nombre, título o ambos). Sin embargo, es relativamente elevado el número de sujetos que recurren exclusivamente al encabezado o al pie de página para indicar sus nombres (un 15,4\% y un $21,7 \%$ de los grupos A y B, respectivamente; véase tabla 17).

Si consideramos correctas, por tanto, las opciones "al comienzo" y "portada", vemos que los hábitos del alumnado principiante contrastan significativamente con el del grupo heterogéneo B (tabla 18):

Tabla 18. Datos identificativos incorrectos

\begin{tabular}{|c|c|c|}
\cline { 2 - 3 } \multicolumn{1}{c|}{} & $\mathrm{N}$ & $\%$ \\
\hline Grupo A & $41 / 52$ & 78.8 \\
\hline Grupo B & $10 / 23$ & 43.5 \\
\hline TotAL (A+B) & $51 / 75$ & 68.0 \\
\hline \multicolumn{2}{|c|}{ Chi $^{2}=9.167$ Sig. 002 } \\
\hline
\end{tabular}

En consonancia con los resultados obtenidos a partir del análisis de las variables anteriores, el número de casos poco adecuados o inadecuados en este caso es demasiado elevado en el grupo A y muy significativo también en el grupo B. A la luz de estos datos, una vez más se deduce cierta desatención ante la correcta presentación de los textos, tanto por parte del alumno como por parte del profesor-evaluador, que no corrige dichos hábitos.

\section{RESUltAdOS GLOBALES}

Si recogemos los datos mostrados anteriormente, observamos que el porcentaje de error en cada uno de los aspectos analizados es alto. La comparación de los dos grupos, A y B, nos muestra, además, que solo tres de los criterios van siendo modificados, en cierto modo, a lo largo de la formación del alumnado: la ubicación de los datos de identificación, el uso de signos de puntuación al final del título y la alineación justificada del texto (gráfico 2): 
Gráfico 2. Porcentajes de error (Grupos A y B)

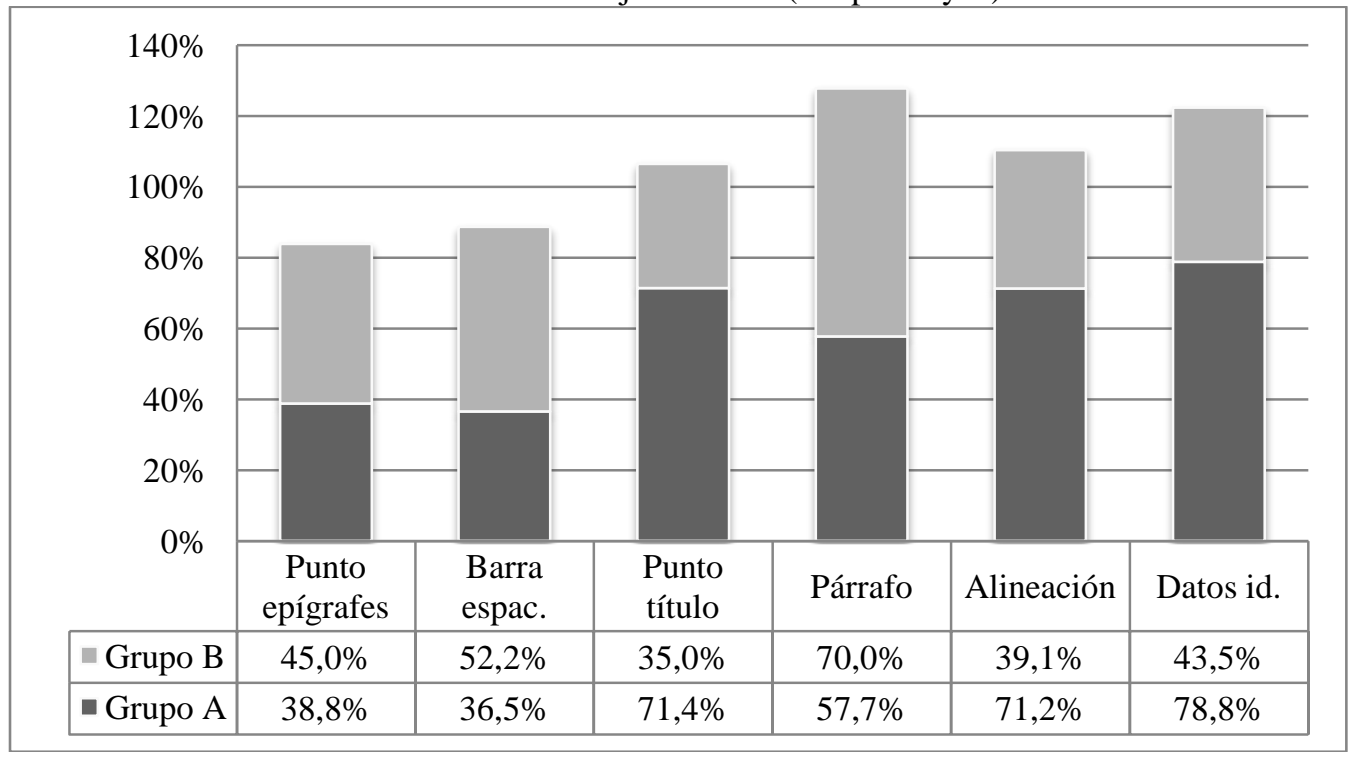

Tras analizar parcialmente cada una de las variables, cabría preguntarse si existe algún sujeto que haya observado, en conjunto, los aspectos para los que existe una norma tipográfica más o menos explícita, esto es, que contemple la norma de corrección de las distintas variables que hemos manejado: justificación, tipo de párrafo (= línea sangrada + separación de párrafos), uso de la barra espaciadora, empleo de signos ortográficos en títulos y epígrafes y reflejo de los datos de identificación.

El resultado, en principio, es negativo: ninguno de los sujetos analizados compone un texto "perfecto" atendiendo a la norma correcta formada por el conjunto de estas seis variables (gráfico 3):

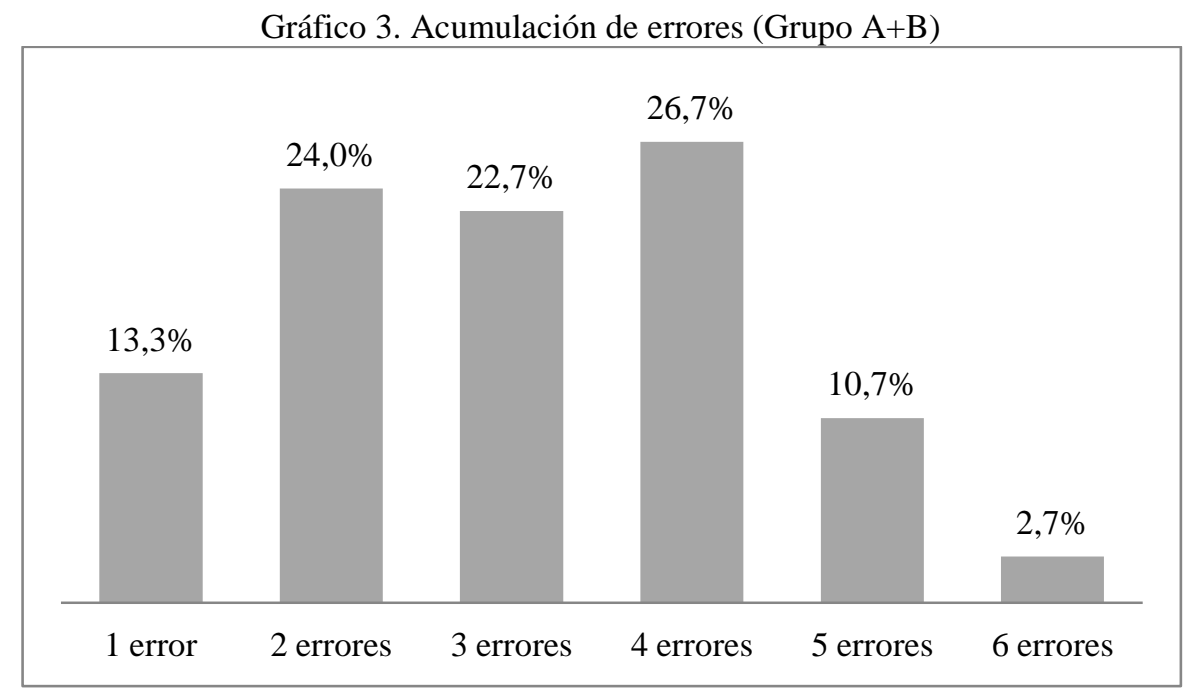

NORMAS. REVISTA DE ESTUDIOS LINGÜÍSTICOS HISPÁNICOS, NÚMERO 1 (AÑO 2011): https://www.uv.es/normas 
Es cierto que no todos los errores aquí tratados reviste la misma gravedad, de modo que al menos un porcentaje mínimo de los textos del corpus podría aproximarse a esa norma de corrección.

Por otra parte, existen diferencias en la distribución de los errores: el más extendido es el de omisión de los datos de identificación, seguido del de la alineación no justificada, la mezcla de criterios en la disposición de los párrafos y el uso del punto en los títulos. Finalmente, encontramos el uso erróneo de la barra espaciadora y el uso de signos en los epígrafes (gráfico 4):

Gráfico 4. Porcentajes de error (Grupo A+B)

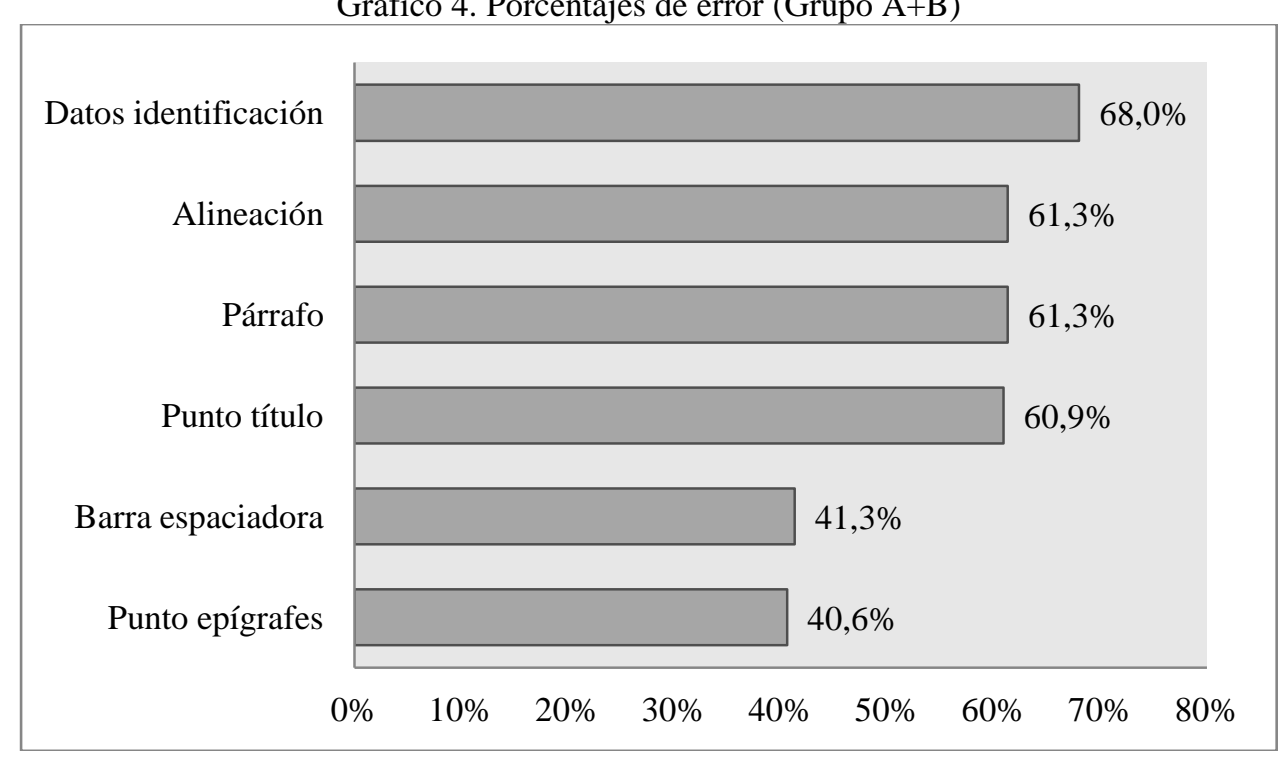

\section{CONCLUSIONES}

Nuestro estudio pone de manifiesto la necesidad de incluir la ortotipografía en el marco de las enseñanzas lingüísticas. La similitud estadística entre el grupo A y el grupo B (de control) en lo que respecta a la mayor parte de los hábitos estudiados denuncia implícitamente un hecho grave: no existe control alguno sobre las prácticas tipográficas y la correcta composición de textos por parte del alumnado a largo de la etapa universitaria, de forma que este sale de las aulas sin apenas nociones sobre los criterios de corrección ortotipográfica para la adecuada presentación de sus escritos en el futuro.

La correcta composición de los escritos suele ser uno de los criterios con los que los docentes evalúan al alumnado universitario. Sin embargo, en el mejor de los casos, esta exigencia suele afectar a la gramática y a la ortografía ${ }^{5}$, pero no a la ortotipografía:

5 Limitamos el concepto de 'corrección' a las perspectivas ortográfica y gramatical; la 'precisión' y la 'propiedad' afectan al plano léxico; la 'adecuación', por su parte, sería el criterio que corresponde a las perspectivas pragmática y textual.

NORMAS. REVISTA DE ESTUDIOS LINGÜÍSTICOS HISPÁNICOS, NÚMERO 1 (AÑO 2011): https://www.uv.es/normas 
no se suele informar al alumno de la mayor conveniencia de unos usos tipográficos frente a otros, ni tampoco se aduce una explicación racional para justificar la preferencia por determinadas elecciones, cuya variedad hace que se perciban muchas veces como caprichosas o puramente personales.

La heterogeneidad tipográfica de los textos producidos por los estudiantes se explica, pues, por un desconocimiento de las normas y de las fuentes normativas sobre este particular, compartido tanto por docentes como por discentes. Se hace necesario, pues, abrir vías para la integración de estas destrezas entre las competencias que ha de haber adquirido el alumnado universitario al final de su etapa formativa.

\section{REFERENCIAS BIBLIOGRÁFICAS}

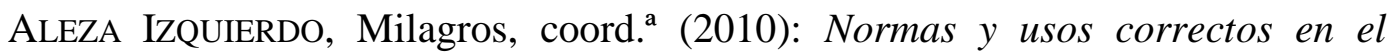
español actual, Valencia, Tirant lo Blanch.

Almela PÉREZ, Ramón (2010): «Textos escritos: pautas de redacción», en Montoro del Arco, Esteban T. y Juan Antonio Moya Corral, eds., El español en contexto, Granada, Editorial Universidad de Granada, 13-38. [En línea]: <http://www.ugr.es/ hum430/Actas_XV_Jornadas.pdf> [consulta: 20/4/2011].

MARTÍNEZ DE SOUSA (2011): «La ortografía académica del 2010: cara y dorso (datos para una recensión)» len línea]: <http://www.martinezdesousa.net/crit_ole2011.pdf> [consulta: 1/5/2011].

MARTÍNEZ DE SOUSA, José (2004): Ortografía y ortotipografía del español actual, Gijón, Trea.

Millán, José Antonio (2008): Manual de urbanidad y buenas maneras en la red, Barcelona, Melusina.

MONTOLÍO DURÁN, Estrella (2010): «La gramática normativa y su importancia en la construcción de la imagen profesional», en Montoro del Arco, Esteban T. y Juan Antonio Moya Corral, eds., El español en contexto, Granada, Editorial Universidad de Granada, 105-119. [En línea]: 〈http://www.ugr.es/ hum430/Actas_XV_Jornadas.pdf> [consulta: 20/4/2011].

MONTOLÍO DURÁn, Estrella, coord. ${ }^{a}$ (2000): Manual práctico de escritura académica, 3 vols., Barcelona, Ariel.

REAl ACADEMia ESPAÑOla (2010): Ortografía de la lengua española, Madrid, Espasa/Real Academia Española.

SHEA, Virginia (1994): Netiquette, San Francisco, Albion Books.

THE OPEN UnIVERSITY, «University E-mail Good Practice Guidance» [en línea]: $<$ http://www.open.ac.uk/university-documents/email-good-practice-guide/> [consulta: 21/4/2011]. 
